\title{
Ruptura de tejidos relacionales: huella de una sinergia corporativa
}

Rupture of relational weaves: footprint of corporate synergy

Rupture de tissus relationnels: trace d'une synergie corporative

\section{Resumen}

Los procesos de implementación de estrategias sinérgicas tienen, inevitablemente, efectos no previstos en los actores sociales de la organización. En este artículo, se presenta la ruptura de tejidos relacionales como un claro ejemplo de estos efectos o huellas ${ }^{1}$, que emergieron al narrar la experiencia individual que los actores sociales tuvieron con la sinergia corporativa en un grupo económico líder del sector de servicios en Colombia. Desde un método cualitativo, una aproximación etnográfica y un enfoque hermenéutico, se exploraron las experiencias y percepciones de diversos actores sociales. La ruptura de tejidos relacionales fue una de las categorías que emergió como

Luz María Rivas Montoya Irivasm@eafit.edu.co Universidad EAFIT Medellín - Colombia

Estudiante del Doctorado de Administración de la Universidad EAFIT-HEC Montreal. Profesora del departamento de Organización y Gerencia en la Escuela de Administración de la Universidad EAFIT.

Artículo de investigación científica y tecnológica Según Clasificación Colciencias

Fecha de recepción: $02 / 02 / 2012$

Fecha de corrección: $03 / 04 / 2012$

Fecha de aprobación: 24/05/2012 huella relevante; esta ruptura se caracteriza por cambios en las relaciones laborales, tensión autonomía/heteronomía y conflictos de poder, situaciones que expresan las consecuencias no previstas de la implementación de la integración de servicios compartidos o sinergia corporativa. Es conveniente advertir que la principal limitación de este estudio exploratorio es que no permite la proposición de modelos ni la construcción de un caso de estudio metodológicamente riguroso. El pensamiento complejo desde la perspectiva de Edgar Morin iluminó el camino de reflexión acerca de la ruptura de tejidos relacionales en cuanto a huella. Estos tejidos son esenciales en cualquier tipo de organización, pero lo son aún más en grupos económicos donde la diversidad de los negocios y culturas exige redes para hacer más productiva y amable la rutina organizacional.

Palabras clave: empresas multinegocio, estrategia corporativa sinérgica, tejidos relacionales.

1. En el presente texto entendemos la palabra huella como los efectos no previstos de la decisión estratégica que impactan a los diferentes actores sociales de la organización según sus percepciones y experiencias. 


\section{Rupture of relational weaves: footprint of corporate synergy}

El capitalismo organizacional: una mirada a la calidad de vida laboral en la docencia universitaria

\section{Rupture de tissus relationnels: trace d'une synergie corporative}

\section{Abstract}

The implementation of processes of synergistic strategies (Ansoff, 1965), inevitably has unforeseen effects on the social organization players. This text presents the relational weave breakdown as a clear example of these effects or traces, which emerged in the storytelling of individual experience that players had with corporate synergy in a leading service sector group in Colombia. From a qualitative method, an ethnographic research strategy and a hermeneutic approach, we explored the experiences and perceptions of various social players. Relational weave breakdown was one of the categories that emerged as a significant footprint; rupture characterized by changes in labor relations, stress autonomy/heteronomy, and power conflicts, situations that express the unintended consequences of implementing shared services integration and corporate synergy. Now it is convenient to note that the main limitation of this exploratory study is that the proposition does not allow constructing models or a methodologically rigorous case study. Complex thinking, from Morin's (1990) perspective, lit the way for reflection on relational weave breakdown. These weaves are essential in any organization, and more so for multi-business groups where the diversity of businesses and cultures requires networks to make more productive and friendly organizational routines.

Keywords: multi business group, synergistic corporate strategy, relational tissue.

\section{Rupture de tissus relationnels: trace d'une synergie corporative}

El capitalismo organizacional: una mirada a la calidad de vida laboral en la docencia universitaria

\section{Rupture of relational weaves: footprint of corporate synergy}

\section{Résumée}

Les processus d'implémentation de stratégies synergiques ont, inévitablement, des effets non prévus chez les acteurs sociaux de l'organisation. Dans cet article, on présente la rupture de tissus relationnels tel qu'un clair exemple de ces effets ou traces, qui ont surgi lors de la narration de l'expérience individuelle que les acteurs sociaux ont eu avec la synergie corporative dans un groupe économique leader du secteur de services en Colombie. Depuis une méthode qualitative, une approximation ethnographique et une approche herméneutique, on a exploré les expériences et perceptions des divers acteurs sociaux. La rupture de tissus relationnels a été une des catégories qui a surgi comme trace éminente ; cette rupture se caractérise par des changements dans les relations de travail, tension autonomie/ hétéronomie et conflits de pouvoir, situations qui expriment les conséquences non prévues de l'implémentation de l'intégration des services partagés ou synergie corporative. II convient d'avertir que la principale limitation de cette étude exploratoire c'est qu'elle ne permet pas la proposition de modèles ni la construction d'un cas d'étude méthodologiquement rigoureux. La pensée complexe depuis la perspective $d^{\prime}$ Edgar Morin a illuminé le chemin de réflexion auprès de la rupture de tissus relationnels quant à la trace. Ces tissus sont essentiels chez $n$ importe quel type d'organisation, mais le sont encore davantage chez des groupes économiques où la diversité des affaires et des cultures exige des réseaux pour faire plus productive et aimable la routine organisationnelle.

Mots clef: entreprises multi affaires, stratégie corporative synergique, tissus relationnels. 


\section{Ruptura de tejidos relacionales: huella de una sinergia corporativa}

\section{Introducción}

Este artículo se focaliza en la ruptura de tejidos relacionales como uno de los hallazgos de la investigación titulada Decisiones estratégicas: una aproximación desde el pensamiento complejo. En este estudio exploratorio se plantea como problema de investigación el carácter limitado y reduccionista de los criterios y referentes de decisión en la visión gerencial tradicional en contraste con una visión compleja de la realidad humana.

Para abordar este problema, se propuso como objetivo general comprender la brecha entre visión gerencial tradicional y visión compleja de la realidad humana, desde la caracterización de criterios y referentes de decisión, a partir de la interpretación de las huellas que las decisiones estratégicas han dejado en los actores sociales.

A su vez, para el logro de este objetivo general, se establecieron los siguientes objetivos específicos: 1 ) Identificar las decisiones estratégicas de la organización consideradas por los actores sociales como más relevantes (alto impacto, alto riesgo y alto consumo de recursos); 2) Explorar opiniones, percepciones y experiencias de estos actores acerca del impacto que dejaron las decisiones consideradas más relevantes; 3) Categorizar las huellas detectadas como las más significativas; y 4) Relacionar los hallazgos y diferentes criterios de decisión para caracterizar la visión gerencial tradicional y contrastarla con los conceptos de complejidad propuestos para identificar las limitaciones de esta visión gerencial.

Para realizar el trabajo de campo, se abordó una organización empresarial donde fuera posible encontrar decisiones estratégicas ${ }^{1}$, es decir, con impacto sobre el largo plazo de la organización y alta demanda de recursos. Por esto se eligió un grupo económico líder en el sector de servicios en Colombia.

La diversidad de perspectivas fue el principal criterio de selección de los actores sociales a entrevistar. Es así como los participantes cumplen con al menos una de las siguientes características: decisores/no decisores; empleados/ex empleados; unidad de negocio/centro corporativo; oficina principal/oficina regional.

Como hallazgos de la investigación están la sinergia ${ }^{2}$, como la decisión estratégica más relevante para los actores sociales; la des-humanización y la ruptura de tejidos relacionales, como las huellas individuales y colectivas percibidas por los entrevistados; el concepto de globalización asumido por otros grupos corporativos relacionados, como principal referente; y eficiencia y conocimiento, como los principales criterios de decisión que aportaron a la rentabilidad esperada por el grupo bajo estudio.

En el presente texto se hace referencia específica a la ruptura de tejidos relacionales en cuanto a huella colectiva. Esta huella expresa el efecto en las relaciones que las personas establecen no sólo para hacer más eficiente su trabajo, sino para tener una mejor calidad de vida laboral.

Esta reflexión se considera relevante porque las estrategias corporativas sinérgicas prometen, según Eisenhardt y Galunic (2000) una mayor generación de valor cuando se administran varios negocios conjuntamente; esta promesa ha desatado un auge por el crecimiento de las empresas a través de fusiones y adquisiciones. Sin embargo, teóricos del campo de la estrategia han presentado diversas dificultades implícitas en las sinergias. Algunas de estas dificultades se relacionan entre otras con la diversidad de los negocios y lógicas dominantes gerenciales (Prahalad \& Bettis, 1986); altos costos de integración (Chaterjee, 2007); y, colaboración entre unidades de negocio (Martin \& Eisenhardt, 2010).

En este sentido, la perspectiva del pensamiento complejo altera los supuestos de análisis organizacionales para responder a la complejidad que caracteriza particularmente a las empresas multinegocio (Chandler, 1962).

1. Las decisiones estratégicas son caracterizadas por varios autores [Mintzberg (1979), Ansoff (1965) y Fredrickson, J. W. (1985)] como aquellas decisiones no rutinarias que involucran un gran compromiso de recursos, cuyas acciones tienen impacto en el desempeño de largo plazo de la organización.

2. Es la manera como se conoce entre los actores sociales la decisión estratégica relacionada con la integración de servicios administrativos. 


\section{Marco teórico y metodológico}

El marco teórico de la investigación se basa en el paradigma de la complejidad, más específicamente, en el pensamiento complejo como método ${ }^{3}$, según los planteamientos de Morin, Ciurana y Domingo (2003). Según Morin (1990) "la complejidad es una palabra problema no una palabra solución” (p. 22) y, en ese sentido, puede aportar a la práctica gerencial y la estrategia corporativa, en tanto que éstas no se enfocan en la solución de problemas concretos, sino más bien, en el diálogo entre situaciones o fenómenos aparentemente opuestos.

Para abordar las decisiones estratégicas desde la perspectiva de los actores sociales, se delimitó el marco de referencia del pensamiento complejo a cuatro pares dialógicos que facilitan el proceso de comprensión de las huellas. Se denominan pares en cuanto refieren dos conceptos aparentemente opuestos y dialógicos en referencia a la imposibilidad de síntesis o solución, lo que suscita un diálogo permanente entre ellos.

\section{Pares dialógicos}

Los pares dialógicos considerados más pertinentes para estudiar las decisiones estratégicas en el contexto empresarial colombiano son causalidad lineal/causalidad recursiva; certidumbre/incertidumbre; Unitas múltiplex; $y$, orden/desorden. A continuación se hará una breve referencia a cada uno de estos.

\section{Causalidad lineal/causalidad recursiva}

Como complemento a la causalidad lineal (o en algunos casos como oposición), la causalidad recursiva es formulada por Morin (1990) como la precursora de las tres causalidades: lineal, circular retroactiva y recursiva. La primera se expresa en un proceso lineal donde "tal cosa produce tal efecto"; en contraste con la linealidad anterior, la recursividad expresa la interdependencia entre productor/producto. En el caso particular de las decisiones, significa que el decisor recibirá las consecuencias de su decisión y, a su vez, la decisión estará influida por las particularidades del decisor.

\section{Certidumbre/incertidumbre}

Hablar de incertidumbre implica azar, aleatoriedad, asimetría, imposibilidad de predicción. Siguiendo las reflexiones de la cibernética frente a la caja negra que existe entre los inputs y los outputs ${ }^{4}$, el pensamiento complejo propone aceptar la incertidumbre como aquella caja indescifrable. Esto significa que al reconocer la imposibilidad de conocer la totalidad del contenido de la caja, habrá nuevas posibilidades de generación de conocimiento. En relación con el proceso decisorio, se considera pertinente recordar la diferencia que hace Morin (1990) entre la posibilidad de una conducción automática de situaciones normales y la necesidad de una estrategia cuando aparece lo inesperado, lo incierto (p. 118). En otras palabras, los criterios de decisión estratégicos no pueden ser previstos anticipadamente, por las situaciones de incertidumbre del entorno.

\section{Unitas múltiplex}

Para el pensamiento mecanicista de Occidente, el todo es igual a la suma de las partes; para el pensamiento sistémico, el todo es más que la suma de las partes, y para el pensamiento complejo, el todo es más y al mismo tiempo menos que la suma de las partes (Unitas múltiplex). Según Morin, lo más difícil es comprender la unidad de lo múltiple y la multiplicidad de lo uno (2003, p. 73).

Najmanovich5 (1993) se apoya en las metáforas del reloj y la red para explicar el concepto de Unitas múltiplex. El reloj ejemplifica el pensamiento mecánico, que puede ser armado y desarmado hasta su última parte, sin alterar su funcionamiento después del rearme. Este plan-

3. Maldonado se refiere a la complejidad como una forma de racionalidad desde tres caminos diferentes pero articulados: la complejidad como método, la complejidad como cosmovisión y la complejidad como ciencia. La primera hace referencia al pensamiento complejo de Morin (Francia); la segunda, al planteamiento de la Escuela de Palo Alto (Estados Unidos), con Bateson como mayor representante, y la tercera, "la complejidad como ciencia es, de lejos, la rama más amplia e importante, y está representada por nombres como I. Prigogine, H. Maturana y F. Varela, S. Kauffman, P. Bak, CH. Langton y otros” (Maldonado, 1999).

4. Los inputs hacen referencia a las entradas en un proceso lineal, mientras que los outputs se refieren a las salidas o productos del proceso. Por ejemplo, para el caso de un proceso de producción, se entienden los inputs como las materias primas y los outputs como el producto terminado.

5. Epistemóloga, PhD de la Universidad Católica de San Pablo, Brasil. 
teamiento de la física mecánica, fue desbordado por la física cuántica ${ }^{6}$ al presentar el principio de indeterminación. En cuanto a la metáfora de la red, esta autora afirma: "Todos participamos de distintas redes y éstas no son sino organizaciones de interacciones, cuyos nodos pueden ser lo que habitualmente llamamos personas, partículas, información...” (Najmanovich, 1993, p. 17).

De otra parte, el ser humano como unidad compleja, es la mejor expresión del Unitas múltiplex. Morin permite reflexionar al respecto al afirmar que "Lo particular se hace abstracto cuando está aislado de su contexto, aislado del todo del que forma parte. Lo global se hace abstracto cuando es sólo un todo separado de sus partes [...]" (2003, p. 195). En referencia a las decisiones estratégicas, este principio resalta la interdependencia individuo/organización, donde las decisiones que tomen los individuos, necesariamente afectarán la organización y a su vez, las decisiones organizacionales impactarán a los individuos. Otra manera de interpretarla en el marco organizacional, es comprender que cada miembro de la organización está influido y, al mismo tiempo, influye las rutinas organizacionales.

\section{Orden/desorden}

La exclusión del desorden puede producir consecuencias nefastas para la empresa; el error y el conflicto son expresiones claras de "desorden" que tienden a ser negadas, e incluso, encubiertas con el propósito de cumplir con el orden establecido. Este par dialógico fundamentado en las estructuras disipativas invita a reflexionar "porque cerca del estado de equilibrio las leyes de la naturaleza son universales; lejos del estado de equilibrio las leyes son específicas. Esas inestabilidades exigen un flujo de energía, disipan energía” (Prigogine, 1999, p. 3).

Es así como la integración teórica que hace Morin entre las estructuras disipativas de Prigogine, la organización viva de von Neumann, el order from noise principle de von Foerster y el Hasard organisateur de Atlan (1970), le permiten afirmar "[...] que entre más se desarrollan la organización y el orden, más complejos se vuelven y más toleran, incluso necesitan, el desorden"(Morin, 1981, p. 75). En este sentido, eliminación del orden como privilegio del orden, puede generar limitaciones a la creatividad y por ende, puede afectar la posibilidad de auto-organización de los procesos y re-creación de productos y servicios, que han de ser diferenciados para competir en el mercado. Volviendo al caso de las decisiones, el exceso de orden limita no sólo la generación de ideas y la participación de diversos actores sociales, sino que genera temores para advertir sobre posibles dificultades posteriores.

Estos cuatro pares dialógicos que se presentan separados como estrategia didáctica de explicación, en la realidad organizacional operan como un todo que se vive en la rutina organizacional. Siguiendo a Morin, el Unitas múltiplex permite ver la paradoja de la organización que es a la vez diversidad en la unidad, variedad y redundancia, unicidad y multiplicidad; por tanto es unidad compleja.

La utilización de los pares dialógicos como marco teórico para observar las decisiones estratégicas permitió develar percepciones y experiencias que serían difícilmente visibles desde la perspectiva económica.

\subsection{Metodología}

La emergencia de la ruptura de tejidos relacionales como huella de una sinergia corporativa fue posible gracias al enfoque cualitativo de la investigación que motivó este texto. Como el propósito de la investigación sobre las decisiones estratégicas pretende superar las aproximaciones reduccionistas a las realidades organizacionales, se eligió la hermenéutica de la acción planteada por Ricoeur (2002), porque ésta propone asumir la acción humana como texto, es decir, como una historia o narración susceptible

6. En los primeros años del siglo XX, se fracturan los principios de continuidad de la física clásica, debido al planteamiento del quantum de Max Planck, desde el cual afirmaba que entre dos puntos no existía nada, es decir, se cuestionaba la causalidad local; esto significa que es imposible determinar la trayectoria de una partícula cuántica, lo que se opone al determinismo propio de la continuidad clásica: "El desarrollo de la física cuántica, tanto como la coexistencia entre el mundo cuántico y el mundo macro físico, han conducido en el plano de la teoría y de la experiencia científica, al surgimiento de pares de contradictorios mutuamente excluyentes (A y no-A): onda y corpúsculo, continuidad y discontinuidad, separabilidad y no separabilidad, causalidad local y causalidad global, simetría y ruptura de simetría, reversibilidad e irreversibilidad del tiempo, etc." (Nicolescu, 1996, p. 20). 
de ser interpretada. Asimismo, el pensamiento de Ricoeur permitió conciliar la tensión explicación/ comprensión al afirmar que:

En el plano epistemológico, en primer lugar, diré que no hay dos métodos, el explicativo y el comprensivo. Estrictamente hablando, sólo la explicación es algo metodológico. La comprensión es más bien el momento no metodológico que, en ciencias de la interpretación, se combina con el momento metodológico de la explicación. Este momento precede, acompaña, clausura y, así, envuelve a la explicación. A su vez, la explicación desarrolla analíticamente la comprensión" (2002, p. 165).

Con la intención de resaltar el carácter subjetivo del ejercicio investigativo, se definió como estrategia de investigación la etnografía enfocada referida por Morse (2003, p. 200), como aquella que se focaliza en pequeños grupos y un tema específico. En cuanto a la técnica de recolección de información, se seleccionó la entrevista abierta por su bajo grado de directividad por parte del entrevistador, que facilita o permite que el protagonismo se centre en el entrevistado. De acuerdo con Delgado y Gutiérrez, este tipo de entrevista "tiende a resultar muy productiva para el estudio de casos típicos o extremos [...] pues tiende a producir una expresión individual (p. 237).

Con la diversidad de perspectivas como criterio de selección de los participantes, se realizaron 24 entrevistas a quienes cumplían al menos a una de las siguientes características: decisores/ no decisores; empleados/ex empleados; unidad de negocio/centro corporativo; oficina principal/ oficina regional. Al finalizar cada entrevista, se diligenció una ficha de observación por parte de la investigadora, donde se consignaron las palabras, detalles, expresiones o percepciones que llamaron especialmente su atención.

Se analizaron a profundidad las 24 fichas de observación obtenidas y 10 transcripciones de entrevistas que representaban las diversas perspectivas referenciadas. Para la técnica de análisis, se inició con la codificación abierta, luego con la axial y la selectiva y se integró el análisis con la codificación por proceso. Este ejercicio de diferentes tipos de codificación se realizó con la ayuda de la herramienta Atlas $t i$, software que apoya la construcción teórica propuesta por la Teoría Fundada en Datos ${ }^{7}$ (Strauss y Corbin, 2002).

Tal como se advirtió antes, en el presente texto se hace referencia específica a la ruptura de tejidos relacionales como huella que ha dejado la sinergia. En este sentido, las decisiones estratégicas pierden su posición protagónica para ceder el énfasis al impacto sentido en las relaciones laborales.

\section{Rupturas de tejidos relacionales}

El concepto de tejido relacional se utiliza en referencia con las relaciones de trabajo, formales e informales, que necesariamente se dan en una organización empresarial. La idea de ruptura alude a la destrucción o anulación de relaciones entre colaboradores de las diferentes unidades de negocio que conforman el grupo empresarial bajo estudio, como consecuencia de la integración de los servicios administrativos.

Expresiones recurrentes sobre aislamiento, discriminación y exclusión son evidencias del rompimiento de las relaciones establecidas durante años, de manera intencional o no, para hacer más fácil, agradable y eficiente su trabajo, es decir, para disfrutar de una buena calidad de vida laboral. Este tipo de experiencias y percepciones fueron evidentes en el momento de la integración de los servicios administrativos; la creación del centro corporativo implicó una reestructuración que, además de incertidumbre, generó confusión por la pérdida de contacto con los interlocutores considerados "válidos".

Según algunos de los entrevistados, los nuevos interlocutores sólo podían ser identificados después de contactar cuatro o más personas; incluso, en varias oportunidades, la respuesta no se obtenía de manera oportuna y además ese interlocutor, por lo general no conocía los requerimientos y características de cada unidad de negocio, lo que implicaba que el corporativo no podía apoyar a la unidad de la manera como se había propuesto.

La desconfianza y poca credibilidad en los nuevos procesos corporativos, así como la presión por la homologación y adaptación, 
generaron, entre otras cosas, resistencia y/o resignación. Relaciones laborales caracterizadas por el colegaje, comenzaron a tomar visos de rivalidad y el nuevo centro corporativo parecía más un conjunto de subgrupos trabajando aisladamente que un centro de servicios implementando una estrategia sinérgica.
Es así como el tejido relacional de cada una de las organizaciones, cohesionado por la autonomía y los años de interacción, sufrió rupturas por la cesión de personal al centro corporativo, los retiros voluntarios de personal no adaptado, y los retiros o reasignación de algunos, cuyas funciones fueron objeto de reestructuración, tal como se ilustra en la Figura 1.

Figura 1. Caracterización de las rupturas de tejidos relacionales como huella

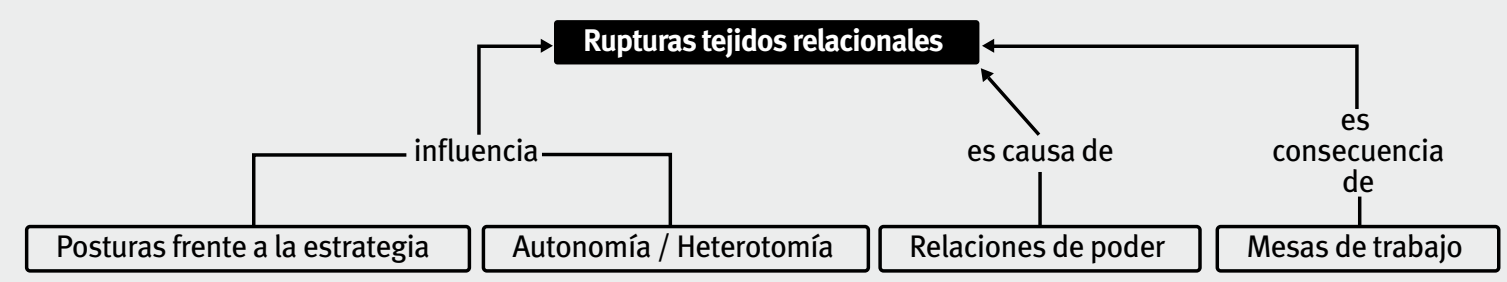

Fuente: Rivas, L. M. (2012).

\subsection{Relaciones de poder}

Parece evidente que la tensión jefe/subordinado es parte de la vida misma en una organización. Parece ser que la relación entre ambos no alcanza el ideal de interdependencia y complementariedad requerido en términos de la eficiencia, sino que por el contrario, el subordinado será siempre visto como una especie de "extraño" necesario, una suerte de "otro" imprescindible pero diferente en la organización. De acuerdo con las reflexiones de Cruz Kronfly, Aktouf y Carvajal (2003), la organización reproduce en su seno el esquema psíquico identitario del "nosotros" y el "ellos", sin poderlo evitar y no obstante la retórica de la igualdad fraternal de sus integrantes, de un modo muy sutil, digamos que incluso inconsciente.

En el caso del grupo bajo estudio, el grado de tensión presenta variaciones significativas a partir de la presión por una mayor productividad y por la necesidad de un reconocimiento que garantizará, de alguna manera, la estabilidad laboral en momentos de cambio continuo. De acuerdo con la opinión de algunos empleados, desde el lineamiento corporativo de hacer seguimiento con indicadores, se ha generado un cambio negativo en la relación jefe/subordinado, porque los indicadores se convierten en la manera como se obtiene el reconocimiento por parte de los superiores, así como mejores oportunidades salariales. La siguiente cita refleja la situación:

Ese tener que cumplir cada uno su indicador hace que se pierda, digamos, la armonía entre jefes y equipo de trabajo, porque cada uno está tan preocupado por mejorar su indicador, y el jefe para que el otro no le dañe su indicador, que hace que la relación sea tensa.

La relación entre compañeros de trabajo también muestra una percepción de deterioro por parte de los entrevistados, sobre todo por situaciones de rivalidad y ausencia de espacios de reunión que fomenten el colegaje. Además, fueron frecuentes las situaciones conflictivas de defensa del territorio, que aparentemente se dan por el énfasis puesto en los resultados. De acuerdo con el testimonio de un ex empleado, la competencia entre sucursales de una de las unidades de negocio, ha llevado a una especie de canibalismo, donde no se respeta el negocio entre asesores ni se piensa en lo mejor para la compañía como grupo:

Yo creo que por eso (énfasis en resultados) es que hay competencia entre sucursales, precisamente por eso, o sea, no lo ven como si fuera la compañía que va a ganar sino que lo ven como la sucursal ganadora, la campeona y empiezan a demostrar obviamente que es la mejor sucursal.

Desde las unidades de negocio, también se expresa la pérdida de relaciones de cooperación entre compañeros de trabajo y la falta de tiempo para debatir acerca de la integralidad del negocio que, anteriormente, fueron en realidad importantes para su evolución. Por el contrario, uno de los entrevistados afirma que ahora las personas que están a cargo de los procesos dan cuenta de un pedacito al otro pedacito, es decir, se han ido creando fronteras que limitan la visión sistémica 
de negocio. Su testimonio permite aclarar la situación:

Ya no ve uno los gobiernos enriquecedores de antes y las críticas no se ven como críticas para construir sino para destruir, hay que tener mucho cuidado en cómo se dicen las cosas, o sea, nos volvimos una organización más formal, menos camaradería, menos colegaje, y eso a mí me ha dado muy duro.

Como otra manifestación del conflicto con las relaciones de poder, se hace referencia al esquema tradicional para toma de decisiones, donde este es un proceso exclusivo de la alta dirección. En este sentido, la posibilidad de discusión o disenso es exclusiva para los niveles altos de la jerarquía, mientras que genera la sensación de exclusión para unos y la sensación de imposición para otros:

Tal vez si yo hubiera tenido la oportunidad de elegir el equipo, porque a este nivel ya si no sé... como hasta el nivel de gerentes, los gerentes pudieron elegir sus equipos, pero a este nivel de directores ya los equipos llegaron con el tiempo...

Uno de los decisores entrevistados, al hacer un análisis en retrospectiva, reconoce el error que suele cometerse en la alta dirección al creer que su trabajo está en el análisis previo a la decisión y en la definición de ésta, pero delegan la responsabilidad de la implementación en otros:

[...] ese es el error que uno comete (en referencia a dejar a otros la implementación de una decisión estratégica), y eso sí pasa en todas partes, la mejor manera es que la gerencia media se apodere, se apropie de la decisión que tú estás tomando, sea complaciente, se identifique plenamente con ella y sienta como que, por lo menos, si no la tomó él, que sienta que es la que él hubiera tomado, pero yo creo que en eso sí, a nosotros nos falta. Nosotros podíamos haber sido un poquito más estratégicos, digamos más bien, no es estratégicos porque la visión no era clara... sino más seguimiento... teníamos el qué, pero no el cómo...

Y precisamente en los cómo, es donde los detalles toman relevancia, y tras las decisiones estratégicas llegan las decisiones rutinarias en las que la visión de la alta dirección puede diluirse fácilmente; en estos casos, la tensión autonomía/ heteronomía se manifiesta de diferentes maneras $y$ en diferentes niveles.

\subsection{Tensión autonomía/heteronomía}

El traslado de las actividades de apoyo desde las unidades de negocio al nuevo centro corporativo, y la consecuente reestructuración, llevó al personal que quedó en las unidades a situaciones de pérdida de poder y de control, pero también para quienes fueron cedidos al corporativo. No es de olvidar que decisiones rutinarias que antes tomaban por sí mismos, o con una consulta sencilla a su jefe directo, se complicaron con la hiperespecialización que caracterizó, en un comienzo, las actividades del centro integrado.

En una de las unidades de negocio, la desconfianza y el descontento con los servicios prestados por el corporativo, se hace evidente en expresiones como:

Yo siento que las áreas de apoyo estaban mucho para que nosotros las apoyemos, no para que ellos nos apoyen a nosotros, no hemos iniciado la fase en la que ellos nos apoyen a nosotros, que es su deber ser en realidad, para que el negocio no pierda, o sea, ganemos porque ahora nos cuesta menos ese tema, pero la ganancia tiene que ir mucho más allá de sólo el resultado en costos [...] mientras más recursos hayan que conozcan el negocio es mucho más fácil la interacción, infortunadamente en la mayoría de esos equipos de trabajo la gente que prevaleció es gente que conoce mucho del tema de seguros.

En el corporativo por su parte, se nota la presión que recibe desde las unidades y la preocupación por "armar ese capital relacional”, tan necesario para que las operaciones fluyan de manera eficaz y eficiente. Al respecto, uno de los entrevistados del corporativo afirma:

Ha sido un proceso como de reconquista de todo el mundo, reconquista para generar confianza, cercanía, en que sientan que es un área que los conoce, porque no generabas ni cinco de credibilidad cuando tú llegas a la ARP y no entendías ni la estructura, no entendías cómo funcionaba una regional, cuáles eran los gerentes estratégicos, los staff, los directivos, los de UEN (Unidades Estratégicas de Negocio), 
los sectores económicos; cuando tú no hablabas como el mismo lenguaje, como que la verdad no generaba confianza.

Otra de las circunstancias que develan las dificultades entre las unidades y el corporativo es la no contextualización, expresada por un empleado de una oficina pequeña, no decisor, en referencia a la exigencia de utilización de proveedores de la oficina central. Su planteamiento es que no siempre la sinergia genera economías de escala, porque el costo de desplazamiento, e incluso de los mismos servicios, es mayor en las ciudades grandes. Su percepción de no contextualización indica que no es posible tomar decisiones localmente y, por el contrario, hay que adaptarse a las definiciones del corporativo.

En estos comentarios, se puede observar la típica tensión entre las unidades de negocio y el centro corporativo, caracterizada por una pérdida de autonomía en las primeras, para pasar a un mayor grado de heteronomía en las actividades de apoyo. En cuanto a las actividades primarias o de negocio, las directivas decidieron realizar pruebas piloto en dos de las ciudades diferentes a la oficina central, pero no fueron implementadas para el total del conjunto corporativo, por lo menos en un primer período.

Conscientes de las dificultades entre corporativo y unidades de negocio, se emprendieron diferentes actividades de capacitación, reuniones de presentación de equipo, viajes a regionales donde una y otra vez recibían la solicitud de tener un interlocutor único. Este proceso se ilustra en el siguiente testimonio:

Armar esa red de relaciones es clave para que esto permee y eso es lo que quieren armar desde marcas, que se empiece como a gestar esa red de relaciones, eso es como una telarañita que se va construyendo con el tiempo, eso no es de la noche a la mañana.

A pesar de esto, después de tres años de retos, aprendizajes, conflictos y tensión entre el centro corporativo y las unidades, pareciera que aún persisten los subgrupos y han sido muchas las personas que se han retirado por no adaptarse al nuevo esquema de trabajo. Uno de los entrevistados planteaba que la sinergia buscaba capitalizar el conocimiento que tenían en las unidades de negocio, pero que ha sido tan duro el proceso de integración y la presión por homologación y adaptación, que mucha gente ha preferido retirarse $y$, por tanto, ha sido necesario volver a generar el conocimiento, sobre todo en las unidades de negocio, situación que, a su parecer, genera mayor vulnerabilidad.

Esta situación invita a reflexionar con Morin para ir más allá del conocimiento como competencia central de una organización...

\begin{abstract}
Aparece entonces la diferencia entre información y conocimiento, porque el conocimiento es organizador. El conocimiento supone una relación de apertura $y$ de clausura entre el conocedor y lo conocido. El problema del conocimiento, así como el de la organización viviente, es el de ser, a la vez, abierto y cerrado. [...] el conocimiento supone no solamente una separación cierta y una cierta separación con el mundo exterior, sino que supone también una separación de sí mismo. Mi espíritu, por más malicioso que sea, ignora todo acerca del cerebro del cual depende (Morin, 1990, p. 152).
\end{abstract}

De esta manera, el retiro voluntario parece ser una decisión equivocada desde un criterio racional; pero otra perspectiva se impuso y el testimonio de personas que se retiraron mostró una realidad menos evidente:

La plata no lo es todo, prefiero estar con tantos pesos menos, un millón, dos millones, lo que sea pero, la tranquilidad...

Entre quienes están aún en la organización, el retiro de personas valiosas también generó desconfianza y temor, porque aparentemente no se tuvo en cuenta la opinión de jefes directos, ni la trayectoria de las personas que se retiraron. Ya para el año 2010, la integración de actividades de apoyo se percibía por algunos como una fase superada:

Ya pasó la tempestad, como que ya nos adaptamos, nos ajustamos.

Pero, para otros, el paso hacia las actividades primarias o de negocio es aún prematuro:

Todavía no nos hemos sentado como negocio integral a definir qué es lo que tenemos que hacer para ganar mercado todos, todavía hay una frontera entre yo manejo esto y ustedes manejan esto... 
De la percepción y experiencias de los entrevistados, se puede deducir el dilema que enfrenta la organización: mantener la cohesión organizacional necesaria para garantizar la productividad y al mismo tiempo alcanzar competitividad en un mercado atractivo para los inversionistas globales. ¿Es posible la solución de este dilema? Este es un claro ejemplo de la imposibilidad de solución o síntesis de los problemas organizacionales cuando se analizan desde una visión compleja, y tal vez sea esa la razón por la que ha prevalecido el reduccionismo de la visión gerencial tradicional.

Pero, ¿qué nos dicen todos estos testimonios? ¿es posible proteger los tejidos relacionales en una organización empresarial inmersa en una sociedad capitalista altamente competitiva?

\section{Reflexiones finales: tejidos relacionales}

Para la construcción de tejidos relacionales fuertes se requiere tiempo, interacción y diálogo. Las rutinas organizacionales favorecen este tipo de interacciones, pero esto no necesariamente implica que se fortalezcan las relaciones laborales. En las relaciones que un sujeto establece con los otros, no solo se "reúnen" sus competencias, habilidades o conocimientos, sino también sus historias de vida. Cada uno lleva consigo las huellas de sus vivencias, sus alegrías y sus tristezas, sus temores y sus expectativas, sus instintos básicos y sus necesidades más específicas.

Cada quien tiene una manera muy particular de observar los fenómenos o situaciones que ocurren en su vida laboral, es decir, cada uno interpreta los hechos de una manera que le permitan encontrar un significado coherente para sí. Hay quienes no alcanzan a percibir esta diversidad de perspectivas, otros las ignoran por su intención de imponer su voluntad, o por la imposibilidad de controlar tanta diversidad. Es este el terreno propicio para la implementación de herramientas de gestión que faciliten el alineamiento de los empleados con la visión organizacional; dicho de otra manera, se anula la diversidad que ofrece la subjetividad.

Este estilo de gestión puede ser útil e incluso eficiente en el corto plazo. Sin embargo, no es sostenible en el tiempo, porque sencillamente en la búsqueda de reconocimiento del sujeto, éste intentará ser diferente, hacerse notar, dejar claro ante sus jefes y compañeros que él es importante para la organización, de manera que ésta pueda garantizarle su estabilidad laboral, es decir, seguridad para sí y para los suyos (en alto grado de certeza).

Creer que existe una organización donde el tiempo y el trabajo conjunto generan un tejido relacional indestructible, es más que una ilusión. La tensión permanente entre individuo y organización, y aún entre los individuos mismos, produce inevitablemente conflictos. Lo importante es valorar esos conflictos, esas tensiones, la diversidad y la pluralidad. Es allí donde nace la creatividad, donde cada uno puede sacar lo peor y lo mejor de sí, donde emerge la diferencia.

Como ese tejido relacional es invisible es poco evidente para muchos; sin embargo, es fundamental para que la información, la comunicación y el trabajo en equipo fluyan. Cuando algún hilo del tejido es retirado, es posible que el tejido se regenere o se reconstruya fácilmente para no alterar su “orden” más allá de lo necesario. Tal como advierte Muñoz (2011), personas y fenómenos sociales, que construyen la esencia misma de la realidad organizacional, son forzados a cambiar a espaldas de sus propias especificidades.

Desde la perspectiva organizacional, la dificultad para la comprensión de la diversidad no justifica la reducción de las perspectivas individuales o, al menos, no sin afectar el compromiso con la organización. Cuando se toma por válida una sola perspectiva, seguramente se alcanzan los resultados y la productividad esperada, pero ¿qué puede ocurrir en el largo plazo? ¿será que no queda más desprotegida una organización sin tejidos relacionales fuertes, frente a las agresiones del entorno y la vulnerabilidad humana?

De esta reflexión se puede considerar que, si bien no es posible mantener tejidos relacionales intactos, sí es fundamental que al menos quienes ostentan el poder (tanto formal como informal) sean conscientes del valor significativo de esos tejidos relacionales tan sutiles y frágiles, pero al mismo tiempo tan poderosos, no sólo en términos de capital sino de transformación de sujetos.

En referencia a las decisiones estratégicas desde la perspectiva del pensamiento complejo habría que decir que, ante la imposibilidad de eliminar su impacto en los individuos, sería deseable considerar, desde otras perspectivas 
que complementan la económica, los sacrificios y dificultades por llegar. Se trata de tener una visión de largo plazo del negocio que permita construir la viabilidad futura de la organización; visión que puede ser nublada por la ilusión que genera la causalidad lineal, la necesidad de certeza, la obsesión por el orden y la negación de la subjetividad.

\section{Referencias}

Ansoff, I. (1965). Corporate Strategy. New York, USA: Mc Graw Hill.

Chandler, A.D. (1962). Strategy and Structure: Chapters in the history of the industrial enterprise. Cambridge, UK: The M.I.T Press.

Chaterjee, S. (2007). Why is synergy so difficult in mergers of related business? Strategy \& Leadership, 35 (2), (pp. 46-52).

Cruz, F., Aktouf, O. y Carvajal, R. (2003). El lado inhumano de las organizaciones. Cali, Colombia: Facultad de Ciencias de la Administración, Universidad del Valle.

Delgado, J. G. (1995). Métodos y técnicas cualitativas de investigación en ciencias sociales. Madrid, España: Síntesis.

Eisenhard, K. \& Galunic, D. (01/01/2001). Coevolving: at last, a way to make synergies work. Harvard Business Review, (p.15).

Maldonado, C. (1999). Esbozo de una filosofía de la lógica de la complejidad. En: Maldonado, C. y Matiz, C. (compiladores).Visiones sobre la complejidad. Bogotá, Colombia: Kimpres.

Martin, J. \& Eisenhardt, K. (2010). Rewiring: crossbusiness unit collaborations in multibusiness organizations. Academy of Management of Journal, 50 (2), (pp. 265-301).

Moreno, J. (2002). Fuentes, autores y corrientes que trabajan la complejidad. En: C. P. COMPLEXUS, ICFES, y UNESCO. Manual de iniciación pedagógica al pensamiento complejo. Bogotá, Colombia: Ediciones Jurídicas Gustavo Ibáñez.

Morin, E. (1984). Ciencia con Consciencia. Barcelona, España: Anthropos.

Morin, E. (1990). Introducción al pensamiento complejo. Barcelona, España: Gedisa.

Morin, E. (1999). Los siete saberes necesarios para la educación del futuro. Medellín, Colombia: UNESCO.

Morin, E., Ciurana, E. y Domingo, R. (2003). Educar en la era planetaria. Valladolid, España: Gedisa.

Morse, J. (2003). Asuntos críticos en los métodos de investigación cualitativa. Medellín, Colombia: Universidad de Antioquia.

Muñoz, R. (2011). Formar en Administración por una nueva fundamentación filosófica. Bogotá, Colombia: Siglo del Hombre Editores, Universidad EAFIT.

Najmanovich, D. (18/09/1993). Del reloj a la red, metáforas para ver al mundo. Futuro, (pp. 12-18).

Prahalad, C. \& Bettis, R. (1986). The dominant logic: a new linkage between diversity and performance. Strategic Management Journal, 7 (6), pp. 485-501.

Prigogine, I. (1999). Filosofía de la inestabilidad. Voprosy Filosofii, (6), (pp. 46-5).

Ricoeur, P. (2002). Del texto a la acción ensayos de hermenéutica II. México, D.F., Mexico: Fondo de Cultura Económica.

Strauss, A. y Corbin, J. (2002). Bases de la investigación cualitativa. Medellín, Colombia: Universidad de Antioquia.

Von Foerster, H. (1998). Por una nueva epistemología. Metapolítica, 8, (pp. 629-641). 